\section{¿QUÉ PASA CON LOS COMITÉS DE ÉTICA DE INVESTIGACIÓN PERUANOS?}

\section{[WHAT HAPPENS WITH THE PERUVIAN ETHICAL REVIEW BOARD?]}

\author{
Salomón Zavala ${ }^{1}$
}

Sr. Editor. En el Perú, durante los últimos cinco años, se han conformado los Comités de Ética de Investigación (CEI) en hospitales públicos, clínicas, universidades y organizaciones no gubernamentales (ONG) hasta alcanzar el número de 30 . Existe entonces una experiencia creciente no solo sobre las tareas que vienen asumiendo sino también en cuanto a los problemas que enfrentan. Nos tomaremos la licencia para comentar sobre alguno de ellos.

La permanencia y desarrollo es un factor importante a considerar, puesto que la tarea que desempeñan los CEI no es fácil y requiere conocimientos, voluntad, tiempo y la experiencia que se adquiere en el ejercicio de la actividad y una permanencia que debería ser de un mínimo de tres años, con una renovación progresiva y parcial. Asimismo, no abundan los profesionales con las características idóneas para la función.

Sin embargo, se ha conocido de actitudes autoritarias y mal uso del poder por parte de dos directores de hospitales públicos que en un caso cambió al presidente de un CEI porque al haber sido elegido presidente del Cuerpo Médico no era grato para el director. En otro caso reciente, los integrantes del CEI se enteraron de manera indirecta que habían sido cambiados sin haberles informado y menos agradecido. Basado en ello, el tratar así a un Comité de Ética, indica que para ser director de un hospital también habrá que exigir un curso básico de ética.

Por otro lado, la capacitación es otro aspecto que debe de considerarse, en ese sentido, el Reglamento de Ensayos Clínicos, exige que los miembros de los CEI tengan Al menos un certificado de capacitación básica en ética en investigación y uno de sus miembros debe tener capacitación en bioética (1). En ese sentido, la Universidad Nacional Mayor de San Marcos ofrece una Maestría en Ética y Bioética Clínica que incluye un módulo de Ética de Investigación. Sin embargo, es difícil que algún miembro de un $\mathrm{CEI}$ se decida a realizar una maestría que dura dos años solo por la parte de

Doctor en Medicina, Instituto de Ética en Salud, Universidad Naciona Mayor de San Marcos. Lima, Perú.

Recibido: 23-08-10 Aprobado: 08-09-10 investigación, aunque en verdad tengo información de dos miembros de un CEI que están interesados.

El costo de la capacitación es un tema que no ha sido abordado adecuadamente hasta la fecha. Si tomamos en cuenta que el trabajo que realizan los miembros de los CEI es voluntario y no remunerado y que el tiempo que exige no solo es de tres a cuatro horas de sesión que se hace como parte de la carga laboral, sino muchas horas más de lectura y estudio de los documentos; el asumir los costos de capacitación por parte de las instituciones donde laboran sería una forma de compensar y asegurar la mejora de estos organismos, efectuándose por medio del uso de los fondos que provienen de los pagos (overhead) que realizan los patrocinadores (sponsors) para la realización de los ensayos clínicos.

En el país, existe la Red de Comités de Ética en Investigación (REDCEI), que ha venido asumiendo decididamente la capacitación de todos los miembros (2), hasta la fecha, 189 han recibido algún tipo de capacitación, 55 han seguido por vía electrónica el Programa de Educación de Ética en Investigación (CITI, de sus siglas en inglés) ${ }^{(3)}$ y 120 han asistido a las actividades realizadas por los Institutos Nacionales de Salud de los Estado Unidos de Norteamérica (NIH).

A los investigadores se les exige entrenamiento sobre buenas prácticas clínicas y los aspectos metodológicos de la investigación, pero no así un curso básico de ética de la investigación. Consideramos que antes de exigirlo, debe existir la posibilidad de hacerlo en el país, además del programa CITI que, dicho sea de paso, un grupo de personas vinculadas con el tema hemos venido revisando y adecuando sus módulos para el Perú.

\section{Conflictos de Interés}

El autor declara no tener conflictos de interés en la publicación de este artículo.

\section{REFERENCIAS BIBLIOGRÁFICAS}

1. Instituto Nacional de Salud. Reglamento de ensayos clínicos. Lima: INS; 2010.

2. Lescano AR, Blazes DL, Montano SM, Moran Z, Naquira C, Ramirez E, et al. Research ethics training in Peru: a cases study. PLoS One 2008;3(9):e3274.

3. Litewka S, Goodman K, Braunschweiger P. El programa CITI: una alternativa para la capacitación en ética de la investigación en América Latina. Acta Bioethica. 2008; 14(1):54-60

Correspondencia: Dr. Salomón Zavala Sarrio

Dirección: Av. Grau 755, Lima01, Perú.

Correo electrónico: szavalas@gmail.com 